\title{
School arrays benefit science as well as students
}

\author{
While cosmic rays arouse interest in physics, more rare phenomena may be spotted.
}

Sir - I read with interest your Editorial on "Schools at $10^{20} \mathrm{eV}$ and beyond" (Nature 429, 685; 2004). We applaud the University of Nijmegen physicists for their success with the high-school project on astrophysics research with cosmics (HISPARC), which has installed cosmic-ray detectors at high schools across the Netherlands.

As the detection rate for these rare highenergy cosmic rays is in the order of one a year per square kilometre of collecting area, it is important to increase the collecting area (by increasing the number of schools involved), and so we welcome HISPARC to a growing family of similar projects across North America and Europe.

In addition, these arrays can search for coincident, nonrandom 'hits' across a large area that could result from correlated primary events from some of the highestenergy objects in the Universe. Again, such phenomena are expected to be very rare and would benefit from the largest collection area possible.

The first cosmic-ray project with an educational dimension was Canada's ALTA project, 'a search in Alberta over a Large area for cosmic-ray shower Time coincidences using an Array of detectors'. This started in 1996 and the idea has been spreading across the world ever since. The ALTA network now consists of 15 sites in high schools across Alberta covering an area of some 100,000 square kilometres.

In addition to searches for cosmic-ray phenomena, the ALTA collaboration of high-school students, teachers and university researchers are pursuing investigations into atmospheric effects on the detection rate for high-energy cosmic rays. Additionally, we will be investigating the use of the arrays to monitor the soft cosmic-ray flux from the Sun - to provide an online 'solar weather report'.
The North American projects are grouped together as the NALTA collaboration (http://csr.phys.ualberta.ca/nalta) and now cover a total collecting area of a few hundred thousand square kilometres. Similar projects in Europe include the Stockholm Educational Air Shower Array (SEASA) in Sweden and Sky-View in Germany. In addition, the Italian Extreme Energy Events (EEE) project is just getting started. A project in the Czech Republic is being jointly developed by the Czech Technical University in Prague and the University of Alberta.

Clearly, more arrays mean more opportunities for students and for science. We would like to welcome HISPARC to this family and wish it continuing success for the future.

James L. Pinfold

Department of Physics, University of Alberta, Edmonton, Alberta T6G 2N5, Canada

\section{Hopes remain for an Alzheimer's vaccine}

Sir - Your News story "Doctors seek lost data on Alzheimer's vaccine" (Nature 430, 715 ; 2004) correctly points out that Elan's approach to treating Alzheimer's disease is "one of the few potentially viable options for controlling the disease". However, we would like to correct an inaccurate statement regarding the results of the discontinued AN-1792 trial.

Research carried out by Nick Fox of the Institute of Neurology in London, presented at the International Conference on Alzheimer's Disease and Related Disorders in July, measured the brain volume of patients enrolled in Elan's clinical trial of AN-1792. Fox found that the brain volume of patients treated with AN-1792 who responded to the treatment by making antibodies diminished by $3 \%$ - not the $6 \%$ stated in your News story and this decrease was only $1 \%$ greater than the brain-volume reduction in those who were treated with a placebo.

In addition, Fox commented that, although the brain-volume reduction was unexpected (not "alarming", as the News story suggests), the small changes seen might be due to the removal of plaque lesions in the brain tissue of patients suffering from Alzheimer's.

We continue to pursue a number of immunotherapy approaches to Alzheimer's disease, including several in preclinical phases of development, with the goal of bringing a meaningful treatment to the millions of individuals, families and caregivers affected by this devastating disease. Dale Schenk

Elan Corporation, 800 Gateway Boulevard, South San Francisco, California 94080, USA

\section{German reforms would make little difference}

Sir - Your Editorial "Weak at the centre" and News story "Court ruling upsets hopes for career reforms" (Nature 430, 593 and 599; 2004) describe the recent failure to replace the traditional career path at Germany's universities, the Habilitation, with a US-style junior-professor system.

It is true that most young German scientists are unhappy with the Supreme Court's decision to stop the reform by declaring it unconstitutional. It is also true that the federal system in Germany, which leaves university policy up to the states, threatens the international competitiveness of German universities. In practice, however, the differences between the existing Habilitation system and the junior professorship are marginal at best.

After completing a $\mathrm{PhD}$ and a postdoc, a Habilitand can join a research group at a German university. Many have no teaching duties and perform independent research, supervising undergraduate and graduate students, writing proposals for grants and publishing as much as they can. A few are well funded by the excellent Emmy Noether programme. Within six years, a
Habilitand has to summarize his or her research achievements: this summary, which is very similar if not identical to most tenure files, is subject to external review. Following an internal university commission, the faculty either grants or rejects the Habilitation; this is similar in many ways to a tenure procedure.

The German academic anachronism is not the Habilitation procedure itself but what follows. Whereas successful US applicants receive tenured positions at their universities, the German Habilitand has to apply for positions elsewhere and sometimes has to wait for years before achieving a tenured associate professorship (C3 status) or a full professorship at another university. In Germany, as elsewhere, the best young scientists will have multiple offers, some before they even finish their Habilitation. But for many others too many - a long, unproductive and insecure phase follows.

Here the junior professorship could indeed help. Unfortunately, it does not, because, as noted in your News story, the German-style junior professorship is not linked to tenure track, forcing junior professors into the same fate as their Habilitand colleagues. Both are waiting and competing for rare openings at other universities. In the end, the differences between the two systems are too small to make much impact on the careers of young German scientists.

\section{Thomas Carell}

Department of Chemistry, University of Munich, Butenandstrasse 5-13, D-81377 Munich, Germany 